\title{
Clinical evaluation of a new rapid fingerstick point- of-care microfluidic immunofluorescence assay for the detection of SARS-CoV-2 antibodies: A prospective evaluation study
}

Jayne E. Ellis ( $\sim$ Jayne.ellis@lumiradx.com )

LumiraDx Ltd

Matthew Morgan

CHPG Primary Care Northglenn

William Simon

PRN of Kansas

Vicki Lawson

LumiraDx Ltd

Nigel M. Lindner

LumiraDx Ltd

\section{Research Article}

Keywords: COVID-19 antibody test, LumiraDx, point of care, SARS-CoV-2, serology test

Posted Date: February 8th, 2022

DOI: https://doi.org/10.21203/rs.3.rs-1268493/v1

License: (c) (1) This work is licensed under a Creative Commons Attribution 4.0 International License.

Read Full License 


\section{Abstract}

Introduction: The LumiraDx SARS-CoV-2 Antibody (Ab) Test is a point-of-care (POC) test that can use fingerstick samples in a rapid, high-sensitivity microfluidic immunofluorescence assay to qualitatively detect total antibodies to severe acute respiratory syndrome coronavirus 2 (SARS-CoV-2). The test was evaluated for clinical agreement using samples with lab-based antibody detection systems and real-time polymerase chain reaction (RT-PCR)-confirmed SARS-CoV-2 infection status across POC settings.

Methods: Plasma samples from participants ( $\geq 18$ years) were collected from clinical studies and commercial suppliers for positive and negative agreement analysis using the Abbott ARCHITECT SARSCoV-2 IgG and RT-PCR as reference tests. Fingerstick application with capillary blood (applied directly or using transfer tube) from participants ( $\geq 18$ years) was analysed following RT-PCR testing and evaluated against Abbott ARCHITECT SARS-CoV-2 IgG, Elecsys Anti-SARS-CoV-2 and RT-PCR test results. Crossreactivity was analysed using samples with known related and high-prevalence pathogens. Application was performed by untrained users and ease of use of the LumiraDx SARS-CoV-2 Ab Test was investigated.

Results: A positive agreement of $100 \%$ (95\% confidence interval [CI]: $92.9,100 \%$; $n=50$ ) was determined in comparison with the Abbott ARCHITECT SARS-CoV-2 IgG test results and 97.2\% (95\% Cl: 90.4, 99.2\%; $\mathrm{n}=72$ ) with RT-PCR, in samples obtained $\leq 118$ days following RT-PCR testing. In participants without a history of SARS-CoV-2 infection, $100 \%$ (95\% Cl: $94.5,100 \% ; n=66)$ negative agreement was established when evaluated against the Abbott ARCHITECT SARS-CoV-2 IgG test and 100\% (95\% Cl: 98.7, 100\%; $\mathrm{n}=290$ ) against RT-PCR testing.

There was $100 \%$ agreement observed between capillary fingerstick application and Abbott ARCHITECT SARS-CoV-2 IgG, Elecsys Anti-SARS-CoV-2 or RT-PCR results, as well as between sample application methods. There was no notable cross-reactivity with any of the tested pathogens and test operators indicated that the LumiraDx SARS-CoV-2 Ab Test was easy to use.

Conclusion: The LumiraDx SARS-CoV-2 Ab Test demonstrated excellent agreement when compared with lab-based antibody detection systems and RT-PCR test results, using capillary whole blood and plasma.

\section{Introduction}

The recent outbreak of severe acute respiratory syndrome coronavirus 2 (SARS-CoV-2), declared a pandemic in March 2020 by the World Health Organization, has caused a global health and economic crisis (1). Owing to the rapid spread of coronavirus disease 2019 (COVID-19), timely and accurate testing for SARS-CoV-2 is a crucial step in disease containment. Detecting SARS-CoV-2 RNA in respiratory tract samples using real-time polymerase chain reaction (RT-PCR) remains the preferred diagnostic test for assessing symptomatic patients with the disease, despite practical limitations such as the need for specialised laboratories and staff, invasive sampling and long turnaround times (2). 
Coronaviruses are a highly diverse family of enveloped positive-sense, single-stranded RNA viruses. The RNA genome is encapsidated by the nucleocapsid $(N)$, and the membrane $(M)$ and envelope $(E)$ ensure its incorporation in the viral particle during the assembly process (3). The spike (S) protein is responsible for recognition and binding to the host cell receptors, such as angiotensin-converting enzyme 2 via the receptor-binding domain (RBD) located on the $\mathrm{S} 1$ domain. This domain is responsible for the fusion and entry of virus into host cells, making it an anticipated target for serology test development $(3,4)$. The detection of an immune response to the virus, as is the case with antibody testing, is an indirect marker of infection and therefore needs to be robust and well validated (5).

The time it takes for patients to seroconvert is dependent on factors such as the patient's immune status and disease severity, with the majority of patients seroconverting by weeks 2-3 following onset of illness (6-8). Currently, antibody levels are most often measured by enzyme-linked immunosorbent assays (ELISA) and chemiluminescence assays (CLIA) (9-12). Although these methods often have high sensitivity and specificity, they require venous sampling, skilled personnel and sophisticated instrumentation. Over the last year, there has been an increasing interest in the use of point-of-care tests (POCTs) in detecting antibodies to SARS-CoV-2 in fingerstick blood samples (5). When conducted by trained personnel, these tests are easy to use, have a rapid turnaround time, and can be performed in remote and rural settings $(8,13)$. Most commercially available SARS-CoV-2 POCTs commonly utilise rapid lateral flow immunoassay technology for the detection of immunoglobulin (Ig)A, IgM and/or $\lg G$ or total antibody to SARS-CoV-2 (13-17).

Globally, over the last few months, various point-of-care (POC) serological assays have come onto the market and have been compared against one another. It is important to note that the reported performance of these POC serological assays differ between countries, either owing to cross-reactivity with pathogens that are prevalent in various geographical regions, a lack of sample cohort, or age, gender and disease severity biases $(14-16,18,19)$. The unsatisfactory performances of some of these POC serological assays may be due to their intrinsic detection limits, which could be suboptimal; sensitivity and specificity are affected by detection methods utilising different SARS-CoV-2 antigens, and thus will have an impact on test performance $(8,11,17)$. Currently, it is unclear whether the presence of SARS-CoV2 antibodies confers immunity against COVID-19 reinfection (20), and for how long antibodies may persist following acute infection is uncertain (13).

SARS-CoV-2 antibody tests can be useful for seroprevalence investigation; in supporting the development of vaccines by providing information, such as extent of infection in a population, and rates of fatality and infection in communities; and in providing information about the antibody response to SARS-CoV-2 after vaccination, such as the development of neutralising antibodies. The LumiraDx SARS-CoV-2 Antibody (Ab) Test is a novel POCT that uses a rapid microfluidic immunofluorescence assay to qualitatively detect total SARS-CoV-2 antibodies (IgA, IgM, IgG) in whole blood, plasma and serum samples (21). The LumiraDx SARS-CoV-2 Ab Test detects antibodies to the S1 domain and the RBD of the virus, which may be associated with neutralising antibody status (22). This study evaluated the clinical performance of the LumiraDx SARS-CoV-2 Ab Test. 


\section{Methods}

\section{Ethical approval}

All included studies had appropriate ethical approval from local Institutional Review Boards and written informed consent from participants was obtained prior to enrolment. In addition, study protocols complied with the Declaration of Helsinki (2013). All participants were aged $\geq 18$ years and were considered as able to donate samples without compromising their current health status.

\section{Clinical validation of the LumiraDx SARS-CoV-2 Ab Test}

\section{Positive and negative agreement compared to the Abbott ARCHITECT SARS-CoV-2 IgG assay}

Validation of the positive $(n=50)$ and negative $(n=66)$ agreement of the LumiraDx SARS-CoV-2 Ab Test was performed via comparison with a commercially available antibody assay, namely the Abbott ARCHITECT SARS-CoV-2 IgG assay (Abbott, Chicago, IL, USA). Study participants were enrolled between 9 July 2020 to 27 August 2020 and showed either symptoms of COVID-19 or were key workers being screened for infection. Capillary blood samples were collected from each participant and tested on the LumiraDx SARS-CoV-2 Ab Test; a plasma sample was obtained from each participant for analysis on the Abbott ARCHITECT SARS-CoV-2 IgG assay at the MRN Diagnostics (Franklin, MN, USA) testing site. Negative agreement was assessed in samples from non-endemic, asymptomatic participants.

\section{Positive and negative agreement compared to RT-PCR testing}

To determine the extent of positive agreement of the LumiraDx SARS-CoV-2 Ab Test in comparison to RTPCR results, plasma samples from a total of 72 participants, who tested positive for SARS-CoV-2 RNA by RT-PCR during the 2019-2020 pandemic, were obtained from commercial suppliers ( $n=22$, Tissue Solutions Ltd, Glasgow, UK; n=50, MRN Diagnostics, Franklin, MA, USA). Plasma samples were collected in anticoagulant tubes containing ethylenediaminetetraacetic acid (EDTA).

To determine the extent of negative agreement, plasma samples (in EDTA-containing tubes) were obtained from 127 participants who tested negative for presence of SARS-CoV-2 RNA via RT-PCR analysis during the 2019-2020 pandemic. This cohort comprised of endemic, non-symptomatic participants ( $n=13$ LumiraDx, Stirling, UK), endemic, symptomatic participants ( $n=15$, LumiraDx clinical study [CAPTURE]; NCT04408066) and non-endemic, asymptomatic participants ( $\mathrm{n}=33$, ZeptoMetrix Corp, Buffalo, NY, USA; $n=66$, MRN Diagnostics). Non-endemic, asymptomatic subject samples collected prior to the 2019-2020 pandemic ( $n=163$ ) were acquired from a UK study (LumiraDx clinical study [NOVEL] (23)) and also from a commercial supplier (MRN Diagnostics). Together, the 290 samples were used to establish the negative agreement between the LumiraDx SARS-CoV-2 Ab Test and RT-PCR testing. 
For assessment of positive and negative agreement, plasma samples were processed and stored at $80^{\circ} \mathrm{C}$ prior to being thawed, mixed, and analysed using the LumiraDx SARS-CoV-2 Ab Test at LumiraDx or approved testing site.

\section{Fingerstick agreement}

In order to investigate equivalence, samples added to the LumiraDx SARS-CoV-2 Ab Test Strip, through direct fingerstick application or via a transfer pipette, were compared against reference assays; however, prior to this, a prospective evaluation was conducted among participants with either a positive or negative SARS-CoV-2 RT-PCR-confirmed test result. Samples applied to the LumiraDx SARS-CoV-2 Ab Test were compared to either RT-PCR, the Abbott ARCHITECT SARS-CoV-2 IgG assay or Elecsys AntiSARS-CoV-2 (Roche, Basel, Switzerland) to assess the level of agreement. Testing on the LumiraDx SARSCoV-2 Ab Test in combination with RT-PCR testing was conducted at five POC sites located in the USA and UK (Eclipse Clinical Research, Tucson, AZ, USA; Professional Research Network of Kansas, Wichita, KS, USA; Centura Health Physician Group, Denver, CO, USA; MRN Diagnostics; LumiraDx). Testing on the LumiraDx SARS-CoV-2 Ab Test in combination with the Abbott ARCHITECT SARS-CoV-2 IgG assay or Elecsys Anti-SARS-CoV-2 test was performed at the MRN Diagnostics (USA) testing site.

All fingerstick samples were from endemic, symptomatic and asymptomatic participants ([CAPTURE-2] NCT04597047 Toolbox CRO; MRN Diagnostics; LumiraDx verification and validation protocol, UK). In addition, equivalence between the two application methods to the LumiraDx SARS-CoV-2 Ab Test, with comparison to the reference assays, was investigated using samples from participants confirmed negative for SARS-CoV-2 infection. Exclusion criteria comprised: participants with skin lesions or conditions that would preclude a fingerstick and/or a venous blood draw; participants who had received

an experimental biological drug, or device, including for treatment or therapy, within 30 days of the visit to the study site; and participants who had previously participated in the current research study.

\section{Cross-reactivity}

Cross-reactivity of the LumiraDx SARS-CoV-2 Ab Test was evaluated by testing a panel of samples positive for antibodies to SARS-CoV-2-related pathogens and high-prevalence disease agents that are reasonably likely to be encountered in clinical specimens and potentially cross-react with the LumiraDx SARS-CoV-2 Ab Test. The test panel comprised plasma or serum samples (Chembio Diagnostics, Hauppauge, NY, USA; Logical Biological, Sandwich, UK) that had tested positive for one of the following: influenza A virus, influenza B virus, hepatitis C virus, hepatitis B virus (genotype D), Haemophilus influenzae, human coronavirus (HCoV) 229E, HCoV-NL63, HCoV-OC43, HCoV-HKU1, anti-nuclear antibody, respiratory syncytial virus, human immunodeficiency virus, mononucleosis, Mycoplasma pneumoniae, Streptococcus pneumoniae, Bordetella pertussis, Mycobacterium tuberculosis and Legionella pneumophila. 


\section{Investigative device}

The LumiraDx SARS-CoV-2 Ab Test is an in vitro diagnostic test intended for professional POC use, composed of a LumiraDx SARS-CoV-2 Ab Test Strip designed to be used in combination with the LumiraDx Platform. The test uses rapid microfluidic immunofluorescence to qualitatively detect total antibodies to SARS-CoV-2 in human whole blood (capillary fingerstick or venous sample), plasma or serum. In short, the LumiraDx Platform is a portable, wall outlet or battery-powered, multi-assay POC instrument. The LumiraDx SARS-CoV-2 Ab Test Strips are single-use, disposable, microfluidic test strips, which contain specific SARS-CoV-2 antigens that can form an immunoassay complex with SARS-CoV-2specific IgM, IgG and IgA antibodies present in whole blood (capillary fingerstick or venous), plasma or serum samples. The test takes approximately 11 minutes to deliver a positive or negative result after the sample has been added to the test strip and inserted into the instrument. The LumiraDx Platform and the LumiraDx SARS-CoV-2 Ab Test Strips are integrated with several quality control checks to ensure the platform and test strips are functioning correctly for each test run (21).

\section{Operator usability study}

The LumiraDx SARS-CoV-2 Ab Test was examined for the ease of use by operators without any prior training at the POC setting. Usability was assessed with seven untrained users across three test sites in the USA, testing a total of 420 participant samples (CAPTURE-2, NCT04597047), and determined via the analysis of errors encountered during the study, ease of recovery from errors and study questionnaire responses. The Intended Use Operator Questionnaire evaluated various metrics of test usability and safety. Responses to each question was rated on a five-point Likert scale, with 1 = strongly disagree and 5 = strongly agree (Figure S1).

\section{Determination of sample size and statistical analysis}

Sample size was determined to meet the minimum Emergency Use Authorization requirements of the US Food and Drug Administration. For this analysis, 30 participants that previously tested positive for presence of SARS-CoV-2 by RT-PCR and 75 participants confirmed negative by the same method were required. In addition, for the fingerstick equivalence study, a minimum of 30 SARS-CoV-2-positive and 30 SARS-CoV-2-negative participants, as confirmed by RT-PCR, were required. Diagnostic performance was evaluated and the results were presented by days since RT-PCR test result. Positive and negative agreement were determined using a Wilson two-sided analysis (24).

\section{Results}

\section{Clinical validation of the LumiraDx SARS-CoV-2 Ab Test compared to reference testing methods}




\section{Positive and negative agreement compared to the Abbott ARCHITECT SARS-CoV-2 IgG assay}

Evaluation of the positive and negative agreement using comparison with the Abbott ARCHITECT SARSCoV-2 IgG assay, showed a positive agreement of 100\% (95\% confidence interval [Cl]: 92.9, 100\%; Table 1) in 50 participants with an RT-PCR documented positive test result for SARS-CoV-2, and $100 \%(95 \% \mathrm{Cl}$ : 94.5, 100\%; data not shown) negative agreement in 66 non-endemic, asymptomatic participants.

Table 1: Positive agreement between the LumiraDx SARS-CoV-2 Ab Test and the Abbott ARCHITECT SARS-CoV-2 IgG assay.

\begin{tabular}{|llll|}
\hline $\begin{array}{l}\text { Days from RT-PCR } \\
\text { test to sample } \\
\text { collection }\end{array}$ & $\begin{array}{l}\text { Number } \\
\text { of } \\
\text { samples }\end{array}$ & $\begin{array}{l}\text { Number of positive samples } \\
\text { detected by the LumiraDx SARS- } \\
\text { CoV-2 Ab Test }\end{array}$ & $\begin{array}{l}\text { Positive agreement with } \\
\text { the Abbott ARCHITECT SARS- } \\
\text { CoV-2 IgG assay }\end{array}$ \\
\hline$\leq 6$ days & 1 & 1 & $100 \%$ \\
\hline $7-13$ days & 0 & NA & NA \\
\hline $14-20$ days & 3 & 3 & $100 \%$ \\
$>21$ days & 46 & 46 & $100 \%$ \\
\hline Total & 50 & 50 & $100 \%$ \\
& & & $(95 \%$ Cl: $92.9,100 \%)$ \\
\hline
\end{tabular}

$\mathrm{Ab}$, antibody; $\mathrm{Cl}$, confidence interval; IgG, immunoglobulin G; NA, not assessed; RT-PCR, real-time polymerase chain reaction; SARS-CoV-2, severe acute respiratory syndrome coronavirus 2.

\section{Positive and negative agreement compared to RT-PCR testing}

Plasma samples from 72 endemic symptomatic participants demonstrated an overall positive agreement of $97.2 \%$ (95\% Cl: 90.4, 99.2\%) between the LumiraDx SARS-CoV-2 Ab Test and SARS-CoV-2 RT-PCR testing. For individuals of whom samples were taken $\leq 6$ days since RT-PCR confirmation, the positive agreement was $84.6 \%$ (Table 2). For those of whom samples were taken between 8-117 days since RTPCR-confirmed infection, the positive agreement was established to be $100 \%$ (Table 2). Plasma samples from a total of 290 endemic, symptomatic and asymptomatic, and non-endemic participants with no history of a positive SARS-CoV-2 RT-PCR test result, showed an overall negative agreement of 100\% (95\% Cl: 98.7, 100\%) between the LumiraDx SARS-CoV-2 Ab Test and SARS-CoV-2 RT-PCR testing (Table 3).

Table 2: Positive agreement between the LumiraDx SARS-CoV-2 Ab Test and RT-PCR test results. 


\begin{tabular}{|c|c|c|c|}
\hline $\begin{array}{l}\text { Days from RT-PCR test to } \\
\text { sample collection }\end{array}$ & $\begin{array}{l}\text { Number } \\
\text { of } \\
\text { samples }\end{array}$ & $\begin{array}{l}\text { Number of positive samples detected by } \\
\text { the LumiraDx SARS-CoV-2 Ab Test }\end{array}$ & $\begin{array}{l}\text { Positive } \\
\text { agreement } \\
\text { with } \\
\text { RT-PCR } \\
\text { testing }\end{array}$ \\
\hline$\leq 6$ days & 13 & 11 & $84.6 \%$ \\
\hline 7-13 days & 7 & 7 & $100 \%$ \\
\hline $14-20$ days & 6 & 6 & $100 \%$ \\
\hline$>21$ days & 46 & 46 & $100 \%$ \\
\hline Total & 72 & 70 & $\begin{array}{l}97.2 \% \\
\text { (95\% Cl: } 90.4, \\
99.2 \%)\end{array}$ \\
\hline
\end{tabular}

$\mathrm{Ab}$, antibody; $\mathrm{Cl}$, confidence interval; RT-PCR, real-time polymerase chain reaction; SARS-CoV-2, severe acute respiratory syndrome coronavirus 2 .

Table 3: Negative agreement between the LumiraDx SARS-CoV-2 Ab Test and RT-PCR test results.

\begin{tabular}{|llll|}
\hline Test population & $\begin{array}{c}\text { Number } \\
\text { of } \\
\text { samples }\end{array}$ & Origin & $\begin{array}{l}\text { Negative agreement with RT- } \\
\text { PCR } \\
\text { test result }\end{array}$ \\
\hline $\begin{array}{l}\text { Endemic, symptomatic participants } \\
\text { (RT-PCR negative) }\end{array}$ & 15 & UK & $100 \%$ \\
\hline $\begin{array}{l}\text { Endemic, asymptomatic participants } \\
\text { (RT-PCR negative) }\end{array}$ & 13 & UK & $100 \%$ \\
\hline $\begin{array}{l}\text { Non-endemic, asymptomatic } \\
\text { participants }\end{array}$ & 99 & USA & $100 \%$ \\
\hline $\begin{array}{l}\text { Non-endemic, asymptomatic } \\
\text { participants }\end{array}$ & 163 & UK/USA & $100 \%$ \\
\hline $\begin{array}{l}\text { Total } \\
\text { nats }\end{array}$ & 290 & UK/USA & $\mathbf{1 0 0 \%}$ \\
\hline
\end{tabular}

$\mathrm{Ab}$, antibody; $\mathrm{Cl}$, confidence interval; RT-PCR, real-time polymerase chain reaction; SARS-CoV-2, severe acute respiratory syndrome coronavirus 2 .

\section{Fingerstick equivalence}

A total of 117 participants were either confirmed positive $(n=62)$ or negative $(n=55$ for SARS-CoV- 2 by RTPCR prior to testing with the LumiraDx SARS-CoV-2 Ab Test, and the Abbott ARCHITECT SARS-CoV-2 IgG assay or the Elecsys Anti-SARS-CoV-2 test. Once determined positive, participants were instructed 
(according to USA State Guidelines) to self-isolate for 14 days before returning for antibody testing with the LumiraDx SARS-CoV-2 Ab Test. Fingerstick specimens from each patient were applied both directly and via a capillary transfer tube. The resulting overall positive agreement was $100 \%$ for both direct and transfer tube fingerstick application to the LumiraDx SARS-CoV-2 Ab Test compared to RT-PCR testing (Table 4). Positive agreement of $100 \%$ was also found between the LumiraDx SARS-CoV-2 Ab Test and the Abbott ARCHITECT SARS-CoV-2 IgG assay or the Elecsys Anti-SARS-CoV-2 (Table 4). The RT-PCRconfirmed positive participants test results, for which the date of the RT-PCR testing was known (48 participants; Table 4), range from 8117 days from the day of RT-PCR testing until the LumiraDx SARSCoV-2 Ab Test was performed (Figure 1).

Table 4: Fingerstick equivalence: Positive agreement.

\begin{tabular}{|c|c|c|c|c|}
\hline $\begin{array}{l}\text { Days } \\
\text { from } \\
\text { RT-PCR } \\
\text { test to } \\
\text { LumiraDx } \\
\text { SARS- } \\
\text { CoV-2 } \\
\text { Ab Test }\end{array}$ & $\begin{array}{l}\text { SARS- } \\
\text { CoV-2 } \\
\text { RT-PCR } \\
\text { result }\end{array}$ & $\begin{array}{l}\text { LumiraDx } \\
\text { SARS-CoV-2 } \\
\text { Ab Test } \\
\text { result / } \\
\text { RT-PCR } \\
\text { result } \\
\text { (\% } \\
\text { agreement) }\end{array}$ & $\begin{array}{l}\text { LumiraDx SARS-CoV-2 Ab Test } \\
\text { result / Abbott ARCHITECT } \\
\text { SARS-CoV-2 IgG assay result } \\
\text { (\% agreement) }\end{array}$ & $\begin{array}{l}\text { LumiraDx SARS-CoV-2 } \\
\text { Ab Test result / Elecsys } \\
\text { Anti-SARS-CoV-2 result } \\
\text { (\% agreement) }\end{array}$ \\
\hline $\begin{array}{l}7-13 \\
\text { days }\end{array}$ & Positive & $2 / 2(100 \%)$ & NA & $2 / 2(100 \%)$ \\
\hline $\begin{array}{l}14-20 \\
\text { days }\end{array}$ & Positive & $2 / 2(100 \%)$ & NA & $2 / 2(100 \%)$ \\
\hline$>21$ days & Positive & $\begin{array}{l}44 / 44 \\
(100 \%)\end{array}$ & $38 / 38$ (100\%) & $44 / 44(100 \%)$ \\
\hline$>14$ days $^{a}$ & Positive & $\begin{array}{l}14 / 14 \\
(100 \%)\end{array}$ & $14 / 14(100 \%)$ & $14 / 14(100 \%)$ \\
\hline Total & Positive & $\begin{array}{l}62 / 62 \\
(100 \%)\end{array}$ & $52 / 52(100 \%)$ & $62 / 62(100 \%)$ \\
\hline
\end{tabular}

${ }^{a}$ The number of days between RT-PCR testing and antibody testing was $>14$ days. However, the exact date the RT-PCR test was performed was not recorded.

Ab, antibody; IgG, immunoglobulin G; NA, not assessed; RT-PCR, real-time polymerase chain reaction; SARS-CoV-2, severe acute respiratory syndrome coronavirus 2 .

Further investigation of the equivalence between sample application methods was performed using samples from individuals who tested negative for SARS-CoV-2 infection. The results revealed no difference between the negative agreement obtained from the LumiraDx SARS-CoV-2 Ab Test and the Abbott ARCHITECT SARS-CoV-2 IgG assay, Elecsys Anti-SARS-CoV-2 or SARS-CoV-2 RT-PCR testing with either direct fingerstick or transfer tube application to the LumiraDx SARS-CoV-2 Ab Test Strip (Table 5). 
Table 5: Fingerstick equivalence: Negative agreement.

\begin{tabular}{|lllll|}
\hline $\begin{array}{l}\text { Sample } \\
\text { application } \\
\text { method }\end{array}$ & $\begin{array}{l}\text { SARS- } \\
\text { CoV-2 } \\
\text { RT-PCR } \\
\text { result }\end{array}$ & $\begin{array}{l}\text { LumiraDx } \\
\text { SARS-CoV-2 } \\
\text { Ab Test } \\
\text { result / } \\
\text { RT-PCR } \\
\text { result } \\
\text { (\% } \\
\text { agreement) }\end{array}$ & $\begin{array}{l}\text { LumiraDx SARS-CoV-2 Ab Test } \\
\text { result / Abbott ARCHITECT } \\
\text { SARS-CoV-2 IgG assay result } \\
\text { (\% agreement) }\end{array}$ & $\begin{array}{l}\text { LumiraDx SARS-CoV-2 } \\
\text { Ab Test result / Elecsys } \\
\text { Anti-SARS-CoV-2 result } \\
\text { (\% agreement) }\end{array}$ \\
\hline $\begin{array}{l}\text { Direct } \\
\text { fingerstick }\end{array}$ & Negative & $\begin{array}{l}53 / 53 \\
(100 \%)\end{array}$ & $15 / 15(100 \%)$ & $40 / 40(100 \%)$ \\
\hline $\begin{array}{l}\text { Fingerstick } \\
\text { via } \\
\text { transfer } \\
\text { tube }\end{array}$ & Negative & $\begin{array}{l}55 / 55 \\
(100 \%)\end{array}$ & $17 / 17(100 \%)$ & $42 / 42(100 \%)$ \\
\hline
\end{tabular}

Ab, antibody; IgG, immunoglobulin G; RT-PCR, real-time polymerase chain reaction; SARS-CoV-2, severe acute respiratory syndrome coronavirus 2 .

\section{Cross-reactivity}

The LumiraDx SARS-CoV-2 Ab Test results showed no notable cross-reactivity when samples positive for antibodies to related pathogens and high-prevalence disease agents were tested (results not shown).

\section{Operator usability at the POC}

During the prospective fingerstick study, untrained users who performed the LumiraDx SARS-CoV-2 Ab Test at POC clinics reported, via a questionnaire, that the test was easy to use. The user error rate across 420 samples was $3.1 \%$. The most common user error encountered was insufficient blood sample added to the test strip. Positive responses from the questionnaire were obtained for following instructions on applying the sample and interpreting the results.

The system was considered easy to use by all users in the study (Figure S1).

\section{Discussion}

This study aimed to evaluate the clinical performance of the LumiraDx SARS-CoV-2 Ab Test in detecting antibodies to SARS-CoV-2 in adults with known COVID-19 status. Moreover, capillary blood sample results from direct fingerstick and transfer pipette application were compared in participants confirmed positive or negative for SARS-CoV-2 by RT-PCR testing and two commercially available 
reference antibody assays; cross-reactivity was assessed and ease of use by untrained operators was analysed.

The LumiraDx SARS-CoV-2 Ab Test, using plasma samples from 362 participants, showed a high level of positive and negative clinical agreement with the standard COVID-19 RT-PCR test, as well as with the Abbott ARCHITECT SARS-CoV-2 IgG antibody assay. The LumiraDx SARS-CoV-2 Ab Test results compared favourably to available data for current lateral flow SARS-CoV-2 antibody POCTs in use, which are generally reported to have low clinical sensitivity $(45-80 \%)$ and specificity $(87-100 \%)(13,15)$. The present study found $100 \%$ positive and negative agreement in detecting SARS-CoV-2 infection in samples collected at least 7 days after COVID-19 confirmation with

RT-PCR test or the Abbott ARCHITECT SARS-CoV-2 IgG test when using the LumiraDx SARS-CoV-2 Ab Test, which agrees with what is known about the timelines of antibody development following illness onset (7). However, even within 6 days of the initial COVID-19 diagnosis, the LumiraDx SARS-CoV-2 Ab Test detected antibodies in $84.6 \%$ of the samples when evaluated against RT-PCR results. This performance is comparable to the available laboratory-based enzyme immunoassays (15), which do not have the advantages of POCTs, such as rapid turnaround time and low-cost detection (8).

Comparison studies of serological assays that have come onto the market have indicated unsatisfactory performances for some of these assays. Different serological tests detect different SARS-CoV-2 antigens (i.e. the S, S1, RBD and N proteins), which affect assay sensitivity and specificity. Validation of different SARS-CoV-2 antigens showed that S1 was more specific in detecting SARS-CoV-2 antibodies than the N protein, resulting in fewer false-positive results (25). The LumiraDx SARS-CoV-2 Ab Test detects all antibodies present in the sample to any region of the RBD and the whole of the S1 domain, which may explain the high negative agreement of the test. The detection of antibodies to both the RBD and the S1 domain, together with microfluidic technology, contribute to the superior positive and negative agreement of the LumiraDx SARS-CoV-2 Ab Test compared with serological assays currently available on the market. Additionally, as it detects antibodies to the RBD and S1 domain, which are the targets for neutralising antibodies, this test has potential for assessing protective immunity by detecting neutralising antibodies, and may therefore play a role in confirming vaccine response (currently under investigation) (26).

Equivalence analyses revealed that the method of fingerstick application did not impact the accuracy of the results, with all samples testing in agreement with the known RT-PCR-confirmed status of the participants. The LumiraDx SARS-CoV-2 Ab Test therefore appears to be flexible enough to use in locations where limited sample collection methods may be feasible (e.g. in rural areas). Analysis of samples positive for antibodies to related pathogens and high-prevalence disease agents that are reasonably likely to be encountered in the clinical specimen, showed no interference, indicating a low risk of false-positive test results due to cross-reactivity.

Although recommended for use by trained personnel, the LumiraDx SARS-CoV-2 Ab Test appeared to have a low error rate even among users who had no prior training and was noted as easy to use by the 
operators. This may be facilitated by the digital, touch-screen interface, which clearly communicates the test result. In contrast, currently available lateral flow POCTs rely on the development of coloured lines, which are often very faint, potentially leading to misinterpretation of the results by inexperienced operatives (15).

One of the limitations of this study was that the data obtained were calculated from the time of

RT-PCR-based confirmation of SARS-CoV-2 status rather than from COVID-19 symptom onset, owing to the symptom onset dates not being accurately recorded. Moreover, it is important to note that to calculate the negative agreement, patient samples from both non-endemic and endemic populations were used, which tested negative for SARS-CoV-2 RNA by RT-PCR. However, because the RT-PCR test only detects the acute presence of SARS-CoV-2 RNA during the first

2-3 weeks following infection, the endemic patient population could have been at risk of having undiagnosed COVID-19, as there is the potential for some participants to be antibody-positive while having received a negative RT-PCR test result. Given that the LumiraDx SARS-CoV-2 Ab Test showed $100 \%$ negative agreement with the RT-PCR test and with the two lab-based antibody detection systems, we do not believe this to have occurred in the current study.

\section{Conclusion}

The results presented in this study indicate that the LumiraDx SARS-CoV-2 Ab Test serves as a highsensitivity, rapid, POC SARS-CoV-2 antibody test that may be used to support SARS-CoV-2 antibody testing using fingerstick samples at point of care testing sites during and after the COVID-19 pandemic. In addition, the LumiraDx Platform has the ability to detect SARS-CoV-2 antigens as well as antibodies in individuals (using the LumiraDx SARS-CoV-2 Antigen Test Strip and LumiraDx SARS-CoV-2 Ab Test Strip, respectively), making this a highly sensitive and effective platform for POC use (27).

\section{Declarations}

\section{Ethics approval and consent to participate:}

All included studies had appropriate ethical approval from local Institutional Review Boards and written informed consent from participants was obtained prior to enrolment. In addition, study protocols complied with the Declaration of Helsinki (2013). All participants were aged $\geq 18$ years and were considered as able to donate samples without compromising their current health status.

\section{Consent for publication:}

Not applicable 


\section{Availability of data and materials:}

The datasets generated and/or analysed during the current study are not publicly available due to commercial agreements but are available from the corresponding author on reasonable request.

\section{Competing interests:}

JEE: Employee of LumiraDx UK Ltd

MM: No conflict of interest

WS: No conflict of interest

VL: Employee of LumiraDx UK Ltd

NML: Employee of LumiraDx UK Ltd

\section{Funding:}

The study was funded by LumiraDx UK Ltd, UK, and Medical Research Network.

\section{Authors' contributions:}

All named authors (JEE, MM, WS, VL, NML) meet the International Committee of Medical Journal Editors (ICMJE) criteria for authorship for this article, take responsibility for the integrity of the work as a whole and have given their approval for this version to be published.

\section{Acknowledgements:}

We thank all the participants of this study, the clinical sites for providing areas to perform the tests, and our research staff and nurses who conducted the study.

Editorial support:The authors acknowledge Maria Haughton, MSci, and Viola Kooij, PhD, of integrated medhealth communication (imc), UK, for medical writing support, which was funded by LumiraDx UK Ltd.

\section{References}

1. World Health Organization. Health emergencies, coronavirus disease (COVID-19) outbreak 2020. Available from: https://www.euro.who.int/en/health-topics/health-emergencies/coronavirus-covid-19 (Accessed February 2021). 
2. Dinnes J, Deeks JJ, Adriano A, Berhane S, Davenport C, Dittrich S, et al. Rapid, point-of-care antigen and molecular-based tests for diagnosis of SARS-CoV-2 infection. Cochrane Database Syst Rev 2020;8:CD013705-CD.

3. V'kovski P, Kratzel A, Steiner S, Stalder H, Thiel V. Coronavirus biology and replication: implications for SARS-CoV-2. Nat Rev Microbiol 2021;19:155-70.

4. Belouzard S, Millet JK, Licitra BN, Whittaker GR. Mechanisms of coronavirus cell entry mediated by the viral spike protein. Viruses 2012;4:1011-33.

5. Theel ES, Slev P, Wheeler S, Couturier MR, Wong SJ, Kadkhoda K. The role of antibody testing for SARS-CoV-2: Is there one? J Clin Microbiol 2020;58:e00797-20.

6. Long QX, Liu BZ, Deng HJ, Wu GC, Deng K, Chen YK, et al. Antibody responses to SARS-CoV-2 in patients with COVID-19. Nat Med 2020;26:845-8.

7. Yongchen Z, Shen H, Wang X, Shi X, Li Y, Yan J, et al. Different longitudinal patterns of nucleic acid and serology testing results based on disease severity of COVID-19 patients. Emerg Microbes Infect 2020;9:833-6.

8. Ricco M, Ferraro P, Gualerzi G, Ranzieri S, Henry BM, Said YB, et al. Point-of-care diagnostic tests for detecting SARS-CoV-2 antibodies: A systematic review and meta-analysis of real-world data. J Clin Med 2020;9:1515.

9. Klüpfel J, Koros RC, Dehne K, Ungerer M, Würstle S, Mautner J, et al. Automated, flow-based chemiluminescence microarray immunoassay for the rapid multiplex detection of IgG antibodies to SARS-CoV-2 in human serum and plasma (CoVRapid CL-MIA). Anal Bioanal Chem 2021;413:561932.

10. Serre-Miranda C, Nobrega C, Roque S, Canto-Gomes J, Silva CS, Vieira N, et al. Performance assessment of 11 commercial serological tests for SARS-CoV-2 on hospitalised COVID-19 patients. Int J Infect Dis 2021;104:661-9.

11. Coste AT, Jaton K, Papadimitriou-Olivgeris M, Greub G, Croxatto A. Comparison of SARS-CoV-2 serological tests with different antigen targets. J Clin Virol 2021;134:104690-.

12. Mekonnen D, Mengist HM, Derbie A, Nibret E, Munshea A, He H, et al. Diagnostic accuracy of serological tests and kinetics of severe acute respiratory syndrome coronavirus 2 antibody: A systematic review and meta-analysis. Rev Med Virol 2021;31:e2181.

13. Robosa RS, Sandaradura I, Dwyer DE, O'Sullivan MVN. Clinical evaluation of SARS-CoV-2 point-ofcare antibody tests. Pathology 2020;52:783-9.

14. Cassaniti I, Novazzi F, Giardina F, Salinaro F, Sachs M, Perlini S, et al. Performance of VivaDiag COVID-19 lgM/lgG Rapid Test is inadequate for diagnosis of COVID-19 in acute patients referring to emergency room department. J Med Virol 2020;92:1724-7.

15. Charlton CL, Kanji JN, Johal K, Bailey A, Plitt SS, MacDonald C, et al. Evaluation of six commercial mid- to high-volume antibody and six point-of-care lateral flow assays for detection of SARS-CoV-2 antibodies. J Clin Microbiol 2020;58:e01361-20. 
16. Montesinos I, Gruson D, Kabamba B, Dahma H, Van den Wijngaert S, Reza S, et al. Evaluation of two automated and three rapid lateral flow immunoassays for the detection of anti-SARS-CoV-2 antibodies. J Clin Virol 2020;128:104413.

17. Vengesai A, Midzi H, Kasambala M, Mutandadzi H, Mduluza-Jokonya TL, Rusakaniko S, et al. A systematic and meta-analysis review on the diagnostic accuracy of antibodies in the serological diagnosis of COVID-19. Syst Rev 2021;10:155-.

18. Kohmer N, Westhaus S, Ruhl C, Ciesek S, Rabenau HF. Brief clinical evaluation of six high-throughput SARS-CoV-2 IgG antibody assays. J Clin Virol 2020;129:104480.

19. Oved K, Olmer L, Shemer-Avni Y, Wolf T, Supino-Rosin L, Prajgrod G, et al. Multi-center nationwide comparison of seven serology assays reveals a SARS-CoV-2 non-responding seronegative subpopulation. EClinicalMedicine 2020;29:100651.

20. World Health Organization. Advice on the use of point-of-care immunodiagnostic tests for COVID-19 2020. Available from: https://www.who.int/news-room/commentaries/detail/advice-on-the-use-ofpoint-of-care-immunodiagnostic-tests-for-covid-19. Accessed February 2021.

21. LumiraDx UK Ltd. LumiraDx SARS-CoV-2 Ab Test 2020. Available from: https://www.lumiradx.com/assets/pdfs/covid-19-antibody-test/sars-cov-2-antibody-ab-productinsert/sars-cov-2-ab-test-strip-product-insert-ous.pdf?v=2. Accessed Feb 2021.

22. Robbiani DF, Gaebler C, Muecksch F, Lorenzi JCC, Wang Z, Cho A, et al. Convergent antibody responses to SARS-CoV-2 in convalescent individuals. Nature 2020;584:437-42.

23. NOVEL Study. Available from: https://www.emergeresearch.org/trial/novel-study. Accessed March 2021.

24. Wilson EB. Probable inference, the law of succession, and statistical inference. J Am Stat Assoc 1927;22:209-12.

25. Sidiq Z, Hanif M, Dwivedi KK, Chopra KK. Benefits and limitations of serological assays in COVID-19 infection. Indian J Tuberc 2020;67:S163-S6.

26. Muecksch F, Wise H, Batchelor B, Squires M, Semple E, Richardson C, et al. Longitudinal serological analysis and neutralizing antibody levels in coronavirus disease 2019 convalescent patients. J Infect Dis 2021;223:389-98.

27. Drain PK, Ampajwala M, Chappel C, Gvozden AB, Hoppers M, Wang M, et al. A rapid, high-sensitivity SARS-CoV-2 nucleocapsid immunoassay to aid diagnosis of acute COVID-19 at the point of care: A clinical performance study. Infect Dis Ther 2021;10:753-61.

\section{Figures}




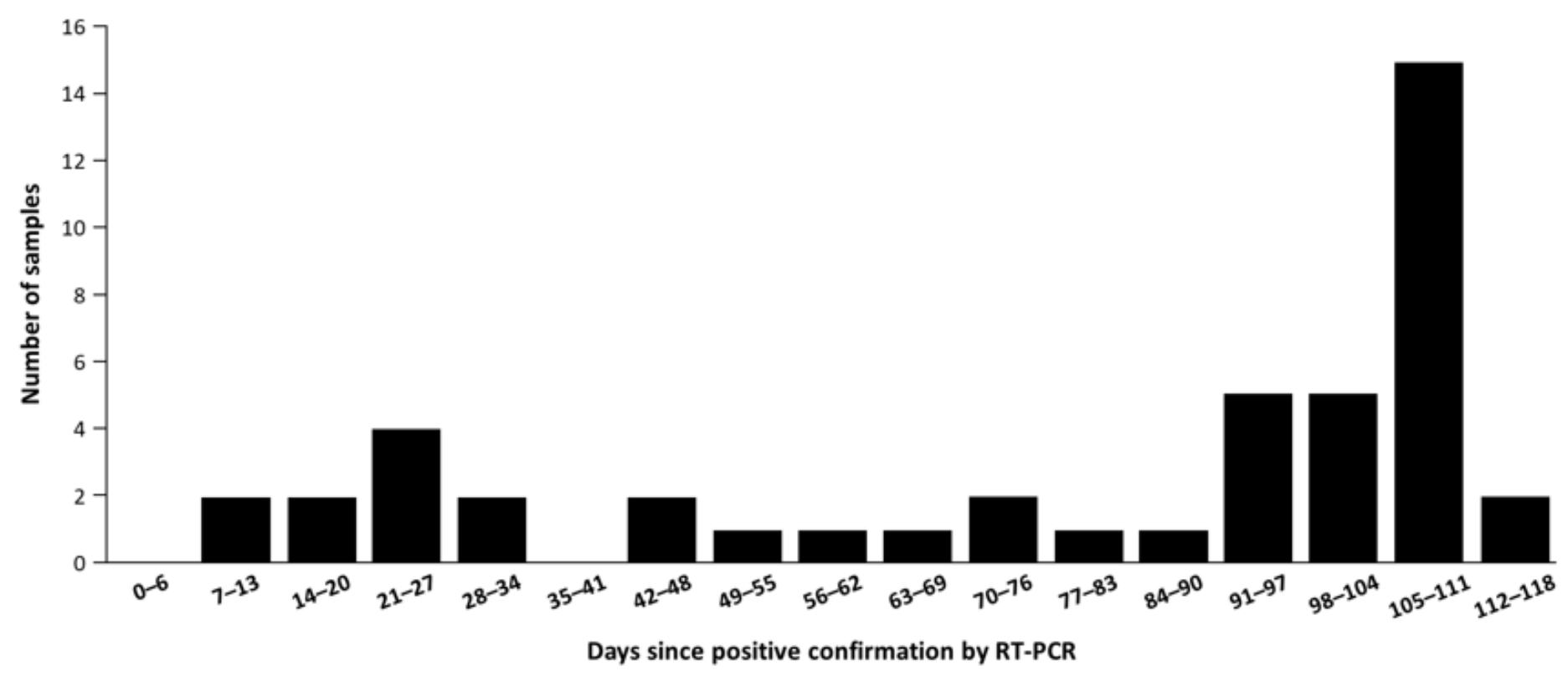

Figure 1

Days between SARS-CoV-2 RT-PCR test and Ab testing for fingerstick analysis. The days on which participants with known positive test results, as confirmed by RT-PCR,

were tested using the LumiraDx SARS-CoV-2 Ab Test ranged from 8 to 117 days.

Ab, antibody; RT-PCR, real-time polymerase chain reaction; SARS-CoV-2, severe acute respiratory syndrome coronavirus 2 .

\section{Supplementary Files}

This is a list of supplementary files associated with this preprint. Click to download.

- s1.png 